\title{
Directional Selectivity through the Subwavelength Slit in Metallic Gratings
}

\author{
Semih Cakmakyapan, Humeyra Caglayan, Andriy Serebryannikov, and Ekmel Ozbay \\ Nanotechnology Research Center, Bilkent University, 06800 Ankara, Turkey \\ Authore-mail address: semihc@bilkent.edu.tr
}

\begin{abstract}
An approach for obtaining strong directional selectivity through a single subwavelength slit in non-symmetric metallic gratings is shown theoretically and experimentally. Directionality effect originates from the different resonance frequencies of two interfaces.

OCIS codes: (050.0050) Diffraction and gratings; (050.2770) Gratings
\end{abstract}

\section{Introduction}

Electromagnetic response of subwavelength apertures has been studied extensively since Bethe showed the theory of diffraction through the subwavelength holes, where the transmitted beam is very low and it is diffracted to every direction [1]. It was shown that the transmitted beam can be enhanced with the aid of periodic corrugations [2]. Optical properties of metallic grating structures can be explained by surface plasmons. A plane or a grooved surface between a Drude metal and dielectric medium can support surface plasmons [3]. Since the skin depth approaches zero for the plane metallic surfaces in the microwave regime, they are not supported by surface plasmons. It was shown theoretically that surface plasmon like modes can be obtained in the perfect electric conductor regime, if there are subwavelength hole arrays on the surface [4]. Later, Hibbins et al experimentally showed that surface plasmon like modes are achievable in microwave frequencies [5]. Coupling the light to the surface plasmons with a periodic structure on the input surface provides enhancement through a subwavelength slit [2]. When the output surface is patterned with periodic gratings, spatial distribution of the transmitted beam can be controlled [6,7]. Surface plasmon based unidirectional transmission has been studied theoretically by using non-symmetric metallic grating structures with one subwavelength slit and double side corrugations [8].

\section{Methods, Experiments and Simulations}

In this letter, we will theoretically and experimentally demonstrate that directional selectivity in transmission through subwavelength slits can be achieved by using metallic slab with corrugated surfaces. Two metallic (Al) grating structures with subwavelength slit at the center are schematically shown in Fig. 1. Slit width is $2 \mathrm{~mm}(\sim \lambda / 10)$ for both structures. The structures are symmetric with respect to the slit center, but the grating periods of the interfaces are different. Grating period of the input surface is $16 \mathrm{~mm}$ for both of the samples, whereas the grating period of output surfaces are $22 \mathrm{~mm}$ and $26 \mathrm{~mm}$ for Sample 1 and Sample 2, respectively.

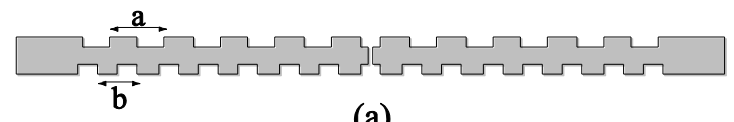

(a)

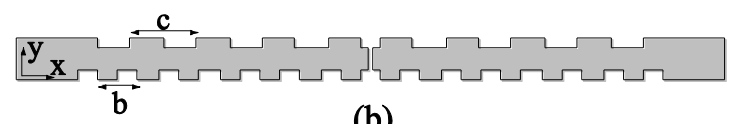

(b)

Fig. 1. Schematic of the metallic (Al) grating structures. (a) Sample 1: $b=16 \mathrm{~mm}$ and $a=22 \mathrm{~mm}$; (b) Sample 2: $b=16 \mathrm{~mm}$ and c=26 $\mathrm{mm}$.

Electromagnetic wave responses of these materials are studied by changing the illumination side, where the structures are illuminated with TM polarized wave (electric field is in the $\mathrm{x}$ direction). Transmission properties over a frequency range are given in Fig. 2. They are calculated and measured $50 \mathrm{~cm}$ away from the slit center. Front-side and back-side of Sample 1 is illuminated with normal incidence. The resonance transmission of Sample 1 is near 11.2 GHz for both front-side and back-side illuminations. Fig. 2 (b) is obtained by tilting the incident beam by 30 degrees. It is seen that the resonance frequencies differ, when different sides of the structure is illuminated. The resonance frequency for the front-side illumination is $9.8 \mathrm{GHz}$, and the back-side illumination shows resonance near $16 \mathrm{GHz}$. Therefore a directional selectivity is obtained. These results can be further understood with angular distributions. 


\section{JTuI67.pdf}

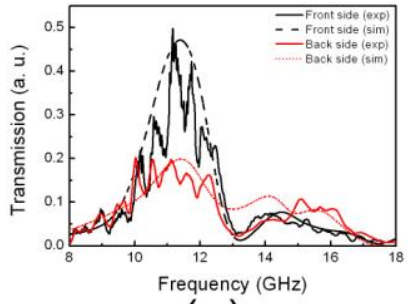

(a)

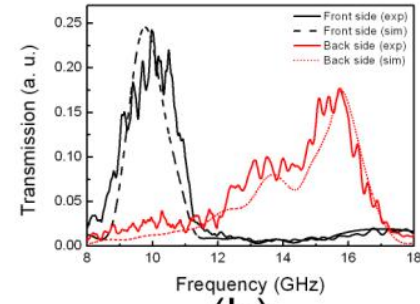

(b)

Fig. 2. Transmission properties of Sample 1 and 2. Observation angle is zero. (a) Sample 1 is illuminated with zero angle of incidence; (b) Sample 2 is illuminated with 30 degree angle of incidence.

Transmission properties of Sample 1 at $11.2 \mathrm{GHz}$ are presented in Fig. 3 (a). For the front-side illumination, we observe beaming at zero-degree observation angle, however, for the back-side illumination, transmission maxima are near \pm 30 degrees. These transmission maxima are analogous to zero-order transmission for the front-side and first-order transmission for the back-side illumination. Furthermore, directional selectivity appears near \pm 30 degrees. Unidirectional transmission at $9.8 \mathrm{GHz}$, which is the resonance frequency of front-side illumination, is observed for Sample 2, and it is demonstrated in Fig. 3 (b). When the structure is illuminated from the front-side, the wave is transmitted at $9.8 \mathrm{GHz}$, and beaming is observed. On the other hand, there is almost no transmitted wave through the slit, when the structure is illuminated from the back-side of Sample 2. The same feature can also be obtained near 16 $\mathrm{GHz}$, which is the resonance frequency of back-side illumination. After a proper optimization these results propose unidirectional devices in the beaming regime, i.e. diode-like devices.

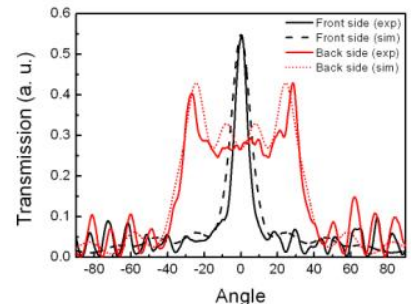

(a)

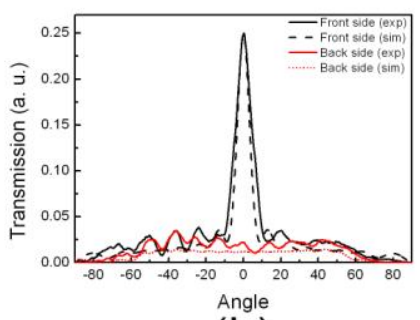

(b)

Fig. 3. Angular distribution of transmission (a) Sample 1; (b) Sample 2

To summarize, we studied the unidirectional beaming through non-symmetric metallic gratings with a subwavelength slit at microwave frequencies. The transmission properties of the structures differ by changing the illumination side, since the surface plasmon resonance of the output surface changes as well.

\section{References}

[1] H. A. Bethe, “Theory of Diffraction by Small Holes," Physical Review 66, 163 (1994).

[2] T. Thio, K. Pellerin, R. Linke, H. Lezec and T. Ebbesen, "Enhanced Light Transmission through a Single Subwavelength Aperture,” Opt. Lett. 26, 1972 (2001).

[3] H. Raether, Surface plasmons on smooth and rough surfaces and on gratings, Berlin, Springer, 1988.

[4] J. Pendry, L. Martin-Moreno and F. Garcia-Vidal, "Mimickinf Surface Plasmons with Structured Surfaces,” Science 305, 847 (2004).

[5] A. Hibbins, B. Evans and J. Sambles, "Experimental verification of designer surface plasmons,” Science 308, 670 (2005).

[6] H. J. Lezec, A. Degiron, E. Devaux, R. A. Linke, L. Martin-Moreno, F. J. Garcia-Vidal, T. W. Ebbesen "Beaming Light from a Subwavelength Aperture,” Science, 297, 820 (2001).

[7] H. Caglayan, I. Bulu, and E. Ozbay, "Beaming of electromagnetic waves emitted through a subwavelength annular aperture," JOSA B, 23, 419-422, (2006).

[8] S. Cakmakyapan, A. E. Serebryannikov, H. Caglayan and E. Ozbay, "One-way transmission through the subwavelength slit in non-symmetric metallic gratings," Opt. Lett. 35, 2597 (2010). 